\title{
EARTH - Energy Aware Radio and Network Technologies
}

\author{
Markus Gruber*, Oliver Blume, Dieter Ferling, Dietrich Zeller, Alcatel-Lucent Bell Labs; \\ Muhammad Imran, University of Surrey, Centre for Communication Systems Research; \\ Emilio Calvanese Strinati, Commissariat à l'Energie Atomique
}

\begin{abstract}
EARTH is a major new European research project starting in 2010 with 15 partners from 10 countries. Its main technical objective is to achieve a reduction of the overall energy consumption of mobile broadband networks by $50 \%$. In contrast to previous efforts, EARTH regards both network aspects and individual radio components from a holistic point of view. Considering that the signal strength strongly decreases with the distance to the base station, small cells are more energy efficient than large cells. EARTH will develop corresponding deployment strategies as well as management algorithms and protocols on the network level. On the component level, the project focuses on base station optimizations as power amplifiers consume the most energy in the system. A power efficient transceiver will be developed that adapts to changing traffic load for an energy efficient operation in mobile radio systems. With these results EARTH will reduce energy costs and carbon dioxide emissions and will thus enable a sustainable increase of mobile data rates.
\end{abstract}

Index Terms - energy efficiency, mobile communication networks, radio access, small cells, energy aware management, load adaptivity, EARTH

\section{INTRODUCTION}

Today it is undisputed that the increasing human emission of greenhouse gases is causing global warming and a reduction is required to limit the temperature rise. The European Commission (EC) has reached in December 2008 an agreement to cut greenhouse gas emissions by $20 \%$ by 2020 and to improve energy efficiency by $20 \%$ [1]. According to the SMART 2020 study [2] the global information and communications technology (ICT) industry accounted for 530 Megatons of $\mathrm{CO}_{2}$ in 2002 and 830 Megatons in 2007. This is approximately $2 \%$ of the global carbon dioxide $\left(\mathrm{CO}_{2}\right)$ emissions and about equivalent to those of global aviation [3]. The contribution of mobile communication networks was $64 \mathrm{Mtons}$ of $\mathrm{CO}_{2}$ in 2002. This may seem a rather small fraction (12\% of ICT emissions) but it is one of the fast growing sectors, expected to grow by nearly a factor of three to 178 Megatons in 2020 (Fig. 1).

The largest fraction of energy consumption occurs in the radio access network, i.e. in the base stations. Compared to this, the energy consumption (and the according $\mathrm{CO}_{2}$

*Contact author: Markus.Gruber@alcatel-lucent.de emissions) of the mobile devices and of the servers in the core network infrastructure each are a factor of 4 or 5 smaller [4].

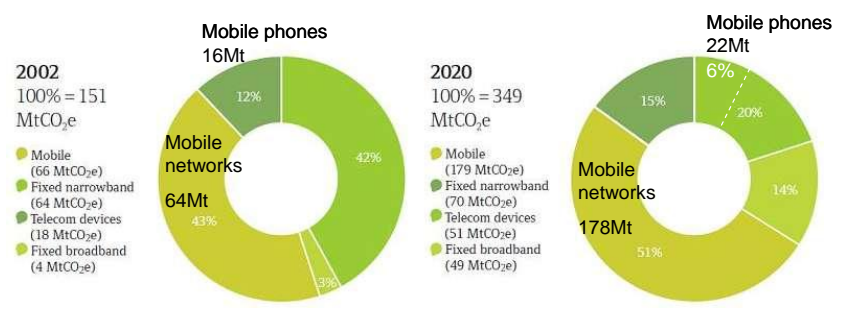

Fig. 1. Contribution of mobile communications (networks and mobile phones) to the $\mathrm{CO}_{2}$ footprint of telecommunication industry in 2002 and estimated for 2020. Source: [2].

With the increasing wireless data rates and the rollout of additional base stations for next generation mobile networks the energy consumption of the network infrastructure is expected to rise even stronger and, at growing energy prices, makes up for a significant fraction of the operational costs of operators. Therefore, energy consumption and $\mathrm{CO}_{2}$ emissions of the mobile network infrastructure have received more and more attention in the telecommunications sector lately. Equipment manufacturers have achieved big progress (fig 2), mainly by increasing the power efficiency of the transceivers in the base stations and by replacing their air conditioning by fresh air cooling [5].

Further improvements of energy efficiency in mobile telecommunications now require more involved research and a holistic approach regarding the entire system as an ensemble.

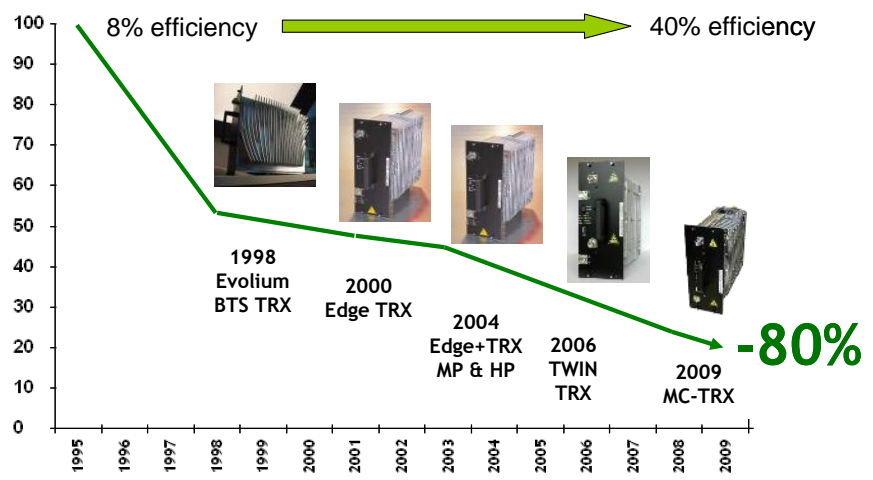

Fig. 2. Continuous improvements of power efficiency in GSM base station transceivers has achieved an $80 \%$ reduction in energy consumption. [5]. 
The European Union has called on the industry to tackle energy efficiency of communication networks and of ICT in general [6] and is funding related research within the framework program FP7. A consortium consisting of 15 partners from industry, academia and SMEs will start the project "Energy Aware Radio and Network Technologies" (EARTH) in January 2010 to find appropriate solutions to this challenge. In this paper, we present the targets of the project and the primary fields of research that appear most promising for potential energy savings.

\section{EARTH AND RELATED WORK}

There has been a significant interest in energy efficiency in ad hoc networks and multihop mesh network architectures due to the limited battery life of the communicating nodes $[7,8,9]$. In conventional cellular communication systems work on the energy efficiency has rather been limited to the mobile terminal with their limited battery power [10], and the energy efficiency of the infrastructure end has been largely ignored. A literature survey for the energy efficiency strategy corresponding to various protocol layers of both infrastructure and ad hoc wireless networks, is available in [11].

There are several recent research projects that consider the issue of energy efficiency of communication systems and their components. OPERA-Net [12] investigates the opportunities to improve the energy efficiency of broadband cellular networks by considering the optimised cooling and energy recovery from the base stations and the optimisation of the components used in communication systems. PANAMA [13], ELBA [14] and Class-S [15] projects focus on techniques of saving energy by more efficient design of the power amplifiers in the communication systems as the major source of energy consumption and typically run at still quite low efficiency. Cool Silicon [16] aims at making recommendations for high performance communication systems with low energy consumption by focusing in three main areas: micro/nano technology, broadband wireless access and wireless sensor networks. Cool Silicon project focuses on the optimisation of individual aspects of the communication systems like the architecture of the system, communication algorithms and protocols and physical components. Mobile VCE Green Radio [17] aims at extending the efficiency studies to the energy metric for cellular and end to end communication.

However, a unified approach to target the whole system from energy efficiency perspective is still missing. Also little effort is devoted to the efficency of next generation wireless acces systems (4G systems).

The new EU FP7 project EARTH is focussing on mobile broadband networks with the future key technologies LTE (3GPP Long Term Evolution) and LTE-Advanced. Its objective is to provide tangible results such as new network architectures and deployment strategies, e.g. with small indoor and outdoor cells, energy aware management mechanisms, and innovative component designs, all with respect to optimised energy efficiency. On top of the already achieved efficiency increase, EARTH has set itself the ambitious goal to further reduce the overall energy consumption of mobile broadband networks by a factor of $50 \%$ and to significantly reduce carbon dioxide emission and operating cost. A major part of the reduction will be reached during low load situations, by ensuring that the energy consumption of access networks is proportional to the traffic load. Eventually, these novelties will be evaluated by simulations and in a mobile network test plant of a major European operator. In the following we will discuss the approaches of EARTH in more detail.

\section{METRICS AND SCENARIOS}

The metrics for comparing different radio access technologies and equipment of different suppliers currently focus on data throughput, spectrum efficiency and consumed power per bases station or component. This neglects different coverage areas, data rates and service level. Finding metrics that are suitable for fairly comparing different solutions is not straightforward, because many different aspects need to be considered (Fig. 3). For instance, a deployment of cells with smaller cell diameters reduces the average path loss of the transmission and thus allows to reduce the transmission power. On the other hand, this requires an increased number of sites with more standby power consumption, more equipment and higher construction effort. As another example, the global energy balance of a mobile phone is not only limited to the energy consumed by the phone when employed by the end user. In a broader sense, it also includes the energy that was spent for production and distribution and is thus implicitly affected by the lifetime of the mobile phone. The lifetime energy balance of end user and of infrastructure equipment substantially differ.

The selection of an appropriate metric becomes even more challenging since it needs to capture the holistic view of energy consumption in the communication system. The techniques adopted to improve the energy efficiency of one end of the communication system (transmitter nodes or receiver nodes) may adversely affect the energy efficiency of the other end. As an example, increasing the frequency reuse in a multiuser system and adopting efficient multiuser sheduling techniques, may reduce the transmit energy requirement for the same spectral efficiency but on the other hand the receiver needs more computation energy for performing multiuser detection. For a fair comparison of any two communication strategies the energy efficiency metric shall encompass not only the transmit energy but also the receiver processing energy and the network operation energy (during service and stand-by states) for the communication strategies under comparison.

Besides analyzing energy balances for different stakeholders individually, EARTH will also elaborate on the global energy balance in order to find environment-friendly solutions that are able to accommodate continuously increasing data rates and is thus committed to have a socio-economic impact and influence on future trends. At the same time it has to be kept in mind that carbon dioxide emissions are not necessarily proportional to the energy consumption of a given system since non-fossil and renewable energy sources are used as well. To achieve the 
more general goal of low energy consumption, however, it is key to know the main consumers of energy in mobile communication systems, which are the base stations and herein the power amplifiers.

The holistic approach EARTH adopts for the definition of metrics is shown in Fig. 4. The analysis covers (from top-tobottom) the very abstract system level down to the more concrete component level. The scope of the very simple and straightforward notion of energy consumption is extended towards competing aspects such as spectral efficiency and quality of service. On the lower layers, physical parameters such as the broadcast of pilot signals and transceiver architectures have to be considered, whereas on higher layers typical scheduling and load balancing behaviours have to be captured.

There are already some efforts going on in standardization bodies on metrics (e.g. $[18,19])$, but it is the target of EARTH to bring all these different metrics together and to reach a reference that is well-accepted in the community. Just like there is a well-accepted driving cycle to measure the fuel consumption of cars in different areas, it is the goal of EARTH to come up with a weighted set of reference scenarios that will be used to assess the efficiency of new solutions in the wireless communications world.

The identified metrics will be used to compare competitive solutions for energy efficiency with each other. Reference scenarios based on typical traffic situations and user distributions in the mobile network will be defined that can later be used to validate the progress made by the project, e.g by deployment strategies with a mix of large and small cells, cooperation schemes such as cooperative multipoint techniques and coordination schemes between different radio access technologies.

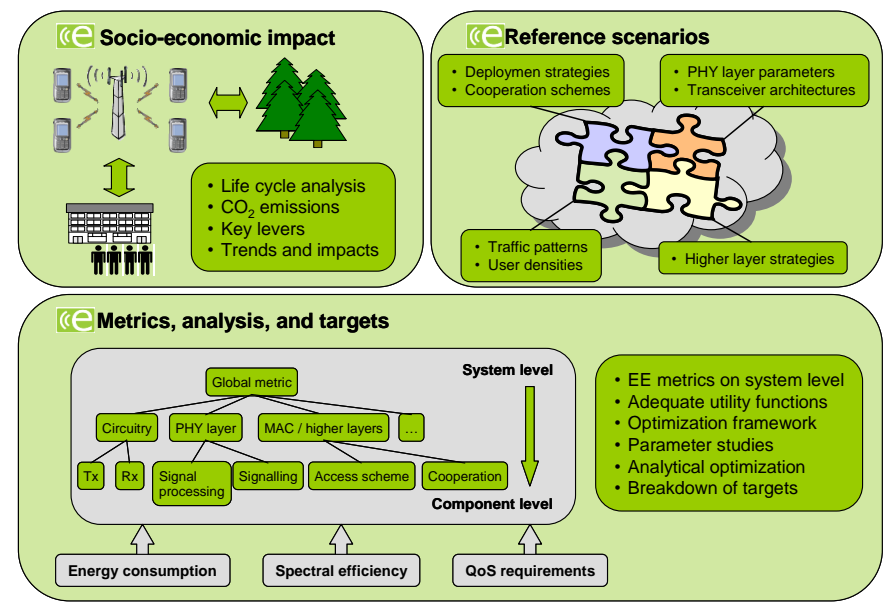

Fig. 3. EARTH's approach on energy efficiency analysis, metrics, reference scenarios and reduction targets.

\section{NETWORK LEVEL}

Energy efficiency optimisation at the network level has been touched in some studies $[20,21]$. However a context in which these studies fit in the overall picture is still missing. Fig. 4 shows the four network level oriented system aspect which the EARTH project focuses on. EARTH targets significant energy savings by using new deployment strategies of radio access networks. Because of the strong decrease in signal strength with respect to the distance of the base station the energy efficiency generally increases with decreasing cell size. This is limited by the fact that each base station must support some basic functionality and that the effort to deploy very large numbers of small base stations may be uneconomical. A crucial task of EARTH will be to determine the ideal cell size that is expected to be significantly smaller than in conventional macro cell deployments. Depending on the reference scenarios discussed before there may even be different ideal cell sizes for different constraints.

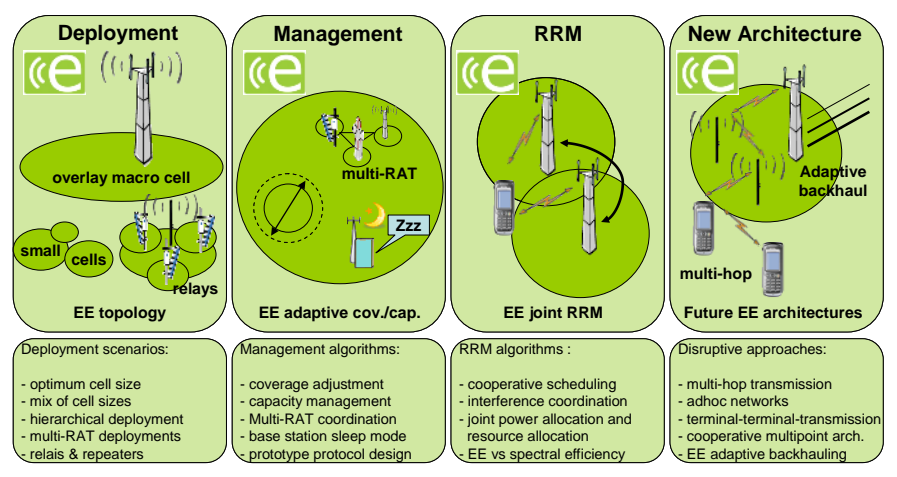

Fig. 4. Green network management tasks in EARTH

Further, the role of repeaters and relays will be analyzed in detail, because these techniques have the inherent advantage that they virtually bring the transmitters closer to the receivers and therefore benefit from the small cell effect discussed above.

An unavoidable consequence of reducing the cell-size or of using repeaters is the stronger inter-cell interference, when the spectrum re-use ratio is kept fixed. Reducing the re-use ratio to control the interference will result in lower spectral efficiency. Alternatives are either to coordinate the interference or to manage it at the receiver end by deploying multiuser detection strategies. These cell cooperation strategies are well studied for their spectral efficiency ([22] and references therein) and can provide starting points to evaluate the energy efficiency of these deployments. As already mentioned, this comes with an associated cost of higher computation energy at the receiver end and in terms of increased energy for control message signalling. A delicate balance should be hold to ensure that the adopted strategy is energy efficient on the overall scale.

Small cell deployments and hierarchical deployments with overlay marco cells may lead to a situation where many cells are scarcely loaded. This particularly applies to situations where the load varies over different times of the day. In high load situations the best solution may be to provide coverage using many small cells, whereas in low load situations cells with only few users can be turned off by network management. Self-organising mechanisms and signalling protocols are required to detect such situations, to redirect the users and to adjust the network coverage, e.g. by change of antenna tilt angles.

EARTH will also analyze and exploit the potential arising from certain radio access technologies being more energy 
efficient for certain types of services than others. From a more algorithmic point of view, radio resource management involves cooperative scheduling and interference coordination algorithms. Also means have to be provided for jointly providing power and radio resources.. Some initial studies on cooperative scheduling [23], power and radio resource management $[20,24,25]$ are available for specific systems and simplified scenarios. It remains to be seen whether absolute limits for quality of service measures are at all appropriate here or what relative decrease in quality of service can be traded off against a decrease in energy consumption without jeopardizing the user experience.

These considerations are not at all limited to conventional deployment options. The project is also committed to identify new architectures with more disruptive approaches such as networks with the possibility of multi-hop transmission or adhoc mesh networks. These approaches benefit from typically small distances from transmitter to receiver and corresponding small transmission powers, but quality of service parameters such as delays cannot be neglected here. Terminal-to-terminal transmissions refer to a scenario where terminals at the cell edge may provide coverage extension to other terminals that fall slightly short of lying within the coverage area of the base station to avoid unreasonably increase of the transmission power at the base station. Finally, other approaches include cooperative multipoint architectures currently being discussed in standardization bodies and adaptive provisioning of backhaul capacities depending on the current needs.

\section{COMPONENT LEVEL}

EARTH will analyze energy saving potentials also on the component level, with respect to the system level aspects of interfaces and network management. As shown in Fig. 5 this follows two main tracks: One is to optimize the components themselves, such as for example the power efficiency of tanscievers, and the other one is to enable power saving measures defined on network level such as a change of the radio frequency band as well as the mobile radio standard by supporting cognitive radio approaches. Specifically, adaptation efforts include power, transmission mode, and load. In any case the major focus of EARTH is to follow an integrated optimization from a holistic point of view and to enable rapid dynamic changes, for instance by providing suitable protocols and signaling means to send components to a standby state.

A huge potential for energy saving is related to the power amplifiers, which consume the largest part of power in the base station (Fig. 6). The undistorted transmission of signals with significant peak-to-average power ratios (PAPR) requires to operate the power amplifiers on average levels way below their maximum signal amplitude. This reducecs the power efficiency, since a simple power amplifier is optimized for maximum output power. The recent evolution of mobile communication systems from GSM to the Long Term Evolution of 3GPP leads to a continuous rise of the PAPR, which will be further increased by using multicarrier and multi-standard solutions.

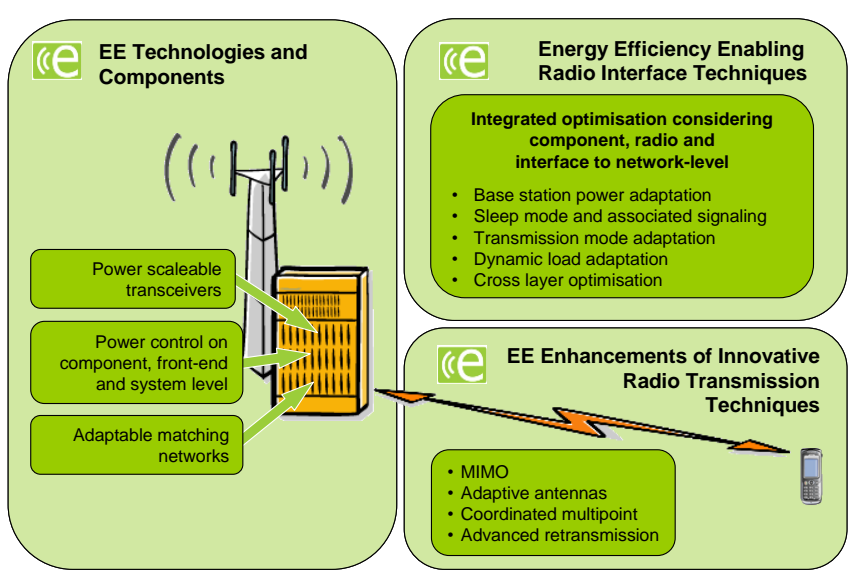

Fig. 5. Green radio

To be up to the requirements of an energy efficient system, future power efficient transceivers need new power amplifier architectures with improved energy efficiency for a certain PAPR combined with measures to reduce this signal parameter. The capability of the transceiver to detect the incoming signal load will allow it to follow energy saving measures taken on system level with a minimum of signaling between different layers. By implementing new interfaces and a certain intelligence, the transceiver can analyze the incoming base band signals and take appropriate actions. For example, the average signal power can be estimated in advance and an adjustment of supply voltages can be perfomed. Also it is possible to switch off some of the several baseband boards or other components.

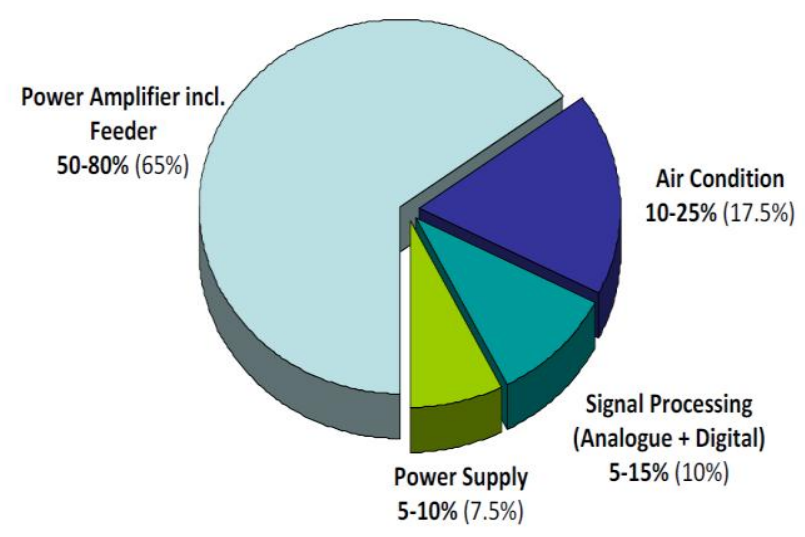

Fig. 6. Breakdown of power consumption in radio base stations [26].

Another source of energy waste are reflections at the antenna back to the transceiver. These reflection depend on the impedance matching of the amplifier, which in turn depends on the power level and the antenna environment. Active tuning of the matching network can cancel these reflections.

Finaly, the interface to the user terminal deserves special attention in the project as there are a number of new transmission techniques that can be exploited for energy efficiency, such as MIMO (multiple input multiple output), adaptive antennas, coordinated multipoint transmission mechanisms, and advanced retransmission techniques. 


\section{CONCLUSION}

Future mobile telecommunication systems have to contribute to reductions in energy consumption and greenhouse gas emissions of ICT. Quite some progress has already been achieved on the the energy efficiency of mobile communication networks, but there is a need for a holistic approach and research on system level innovations. EARTH is a European research project that has set itself the target to provide a holistic analysis of state-of-the-art systems and to develop improvements in order to come up with integrated deployment recommendations for future mobile communication networks. A two-fold approach is pursued in the project: On the network level, architectures, protocols, and algorithms are considered, whereas on the component level strategies to optimize individual elements and their dynamic adaptation to the actual needs are regarded. The ambitious goal of EARTH is to cut the energy consumption of mobile broadband networks by $50 \%$. The findings will be evaluated using in simulations and in a large testbed operated by a major telecommunication operator.

The innovations worked out in EARTH will be combined into an integrated solution. Tangible results will be introduced to standardization bodies and industry exploitation in order to ensure impact of the project to the technical community. This will enable a sustainable deployment of future broadband mobile communication sytems, with respect to carbon dioxide emissions as well as to operational costs. Leveraging these networks, further reductions of the global $\mathrm{CO}_{2}$ footprint are expected by intensive use of ICT services, e.g. for teleconferencing, telecommuting and dematerilisation.

\section{ACKNOWLEDGMENT}

We acknowledge the contribution of the EARTH consortium to the technical definition of the project.

\section{REFERENCES}

[1Climate change: Commission welcomes final adoption of Europe's climate and energy package", EC press release Dec. 2008,

http://europa.eu/rapid/pressReleasesAction.do?reference=IP/08/1998

[2] "SMART 2020: Enabling the low carbon economy in the information age", The Climate Group, Global eSustainability Initiative (GeSI). http://www.smart2020.org/

[3] Gartner Study, 2007. http://www.gartner.com/it/page.jsp?id=503867

[4] “Energy Efficiency in Telecom Networks", Light reading webinar, 2008. http://metacast.agora.com/link.asp?m=51363\&s=4452706\&l=0

[5] Alcatel-Lucent GSM product data. http://www1.alcatellucent.com/tmp_Static/powerperformance/powerperformance.jhtml

[6] "EU Commissioner calls on ICT industry to reduce its carbon footprint by $20 \%$ as early as 2015 ", EC press release: MEMO/09/140, 30/03/2009, http://europa.eu/rapid/pressReleasesAction.do?reference=MEMO/09/140

[7] S. Cui, A. J. Goldsmith, A. Bahai, "Energy-efficiency of MIMO and cooperative MIMO techniques in sensor networks," IEEE J. Sel. Areas Commun., vol. 22, no. 6, pp. 1089-1098, Aug. 2004.
[8] Catovic, S. Tekinay, T. Otsu, "Reducing transmit power and extending network lifetime via user cooperation in the next generation wireless multihop networks," Journal on Communications and Networks, vol. 4, no. 4, pp. 351362, Dec. 2002.

[9] E. Shih et al., "Physical Layer Driven Protocol and Algorithm Design for Energy-Efficient Wireless Sensor Networks," Proc. ACM MobiCom '01, Rome, Italy, July 2001, pp. 272-86.

[10] E Shih, P Bahl, MJ Sinclair, "Wake on wireless: an event driven energy saving strategy for battery operated devices," Proc. 8th annual international conference on Mobile computing and networking, 2002.

[11] C. E. Jones, K. M. Sivalingam, P. Agrawal, J. C. Chen, "A survey of energy efficient network protocols for wireless networks," Wireless Networks, vol. 7, no. 4, pp. 343-358, Aug. 2001.

12] Optimising Power Efficiency in mobile RAdio Networks (OPERA-Net), EUREKA CELTIC project, http://opera-net.org/default.aspx

[13] PANAMA (Power Amplifiers aNd Antennas for Mobile Applications), EUREKA CATRENE project, 2009-2011

[14] Energy Efficiency of linear power amplifiers for mobile telecommunication base stations (ELBA), BMBF research project, Förderschwerpunkt "mobileGaN", 08/2006 - 07/2009. http://www.pt-it.pt-dlr.de/de/1760.php

[15] Class-S, German research project in BMBF Förderschwerpunkt "mobileGaN", 09/2006 - 02/2010. http://www.pt-it.pt-dlr.de/de/1760.php

[16] Spitzencluster COOL SILICON, www.cool-silicon.de/

[17] 'Green Radio - Sustainable Wireless Networks', February 2009, Core 5 Programme of Mobile VCE, Virtual Centre of Excellence in Mobile \& Personal Communications Limited,

http://www.mobilevce.com/dloads-publ/mtg284Item_1503.ppt

[18] "Reduction of Energy Consumption in Telecommunications Equipment and Related Infrastructure", TR 102530 and "Energy Consumption in BB Telecom Network Equipment", TS 102 533, ETSI Environmental Engineering, 2008. http://www.itu.int/net/home/index.aspx

[19] ATIS Green Initiative, 2008. http://www.atis.org/Green/index.shtml

[20] N. Feng, S. C. Mau, and N. B. Mandayam, "Pricing and Power Control for Joint Network-Centric and User-Centric Radio Resource Management," IEEE Trans. Commun., vol. 52, no. 9, pp. 1547-1557, Sep. 2004.

[21] N. Bambos, "Toward power-sensitive network architectures in wireless communications: concepts, issues, and design aspects," IEEE Personal Communications, vol. 5, no. 3, pp. 50-59, Jun 1998.

[22] E. Katranaras, M. A. Imran, C. Tzaras, "Uplink Capacity of a Variable Density Cellular System with Multicell Processing," IEEE Transactions on Communications, vol. 57, no. 7, pp. 2098 - 2108, July 2009.

[23] Z. Han. T. Himsoon, W. P. Siriwongpairat and K.J.R. Liu , "Energyefficient cooperative transmission over multiuser OFDM networks: who helps whom and how to cooperate," in proceedings of IEEE Wireless Communications and Networking Conference, 13-17 March 2005, pp: 1030- 1035

[24] G. Marqués, F. F. Digham, G. B. Giannakis, Optimizing "Power Efficiency of OFDM Using Quantized Channel State Information," IEEE Journal on Selected Areas in Communications, vol. 24, no. 8, august 2006

[25] G Miao, N Himayat, Y Li, D. Bormann, "Energy Efficient Design in Wireless OFDMA," IEEE International Conference on Communications, ICC'08, 19-23 May 2008, pp: 3307-3312

[26] "Energy Efficient Radio Access Network (EERAN) Technologies", Unpublished Study, Alcatel-Lucent and, TU Dresden, Vodafone Chair Mobile Communications Systems, 2009[ 